\title{
Surface pitting on nanofibrous matrix as an ultra- sensitive indicator for enzymatic hydrolysis of crystalline cellulose
}

\author{
Mikiko Tsudome $^{1}$, Mikako Tachioka ${ }^{1}$, Kohsuke Uchimura ${ }^{1, \dagger}$, Miwako Tsuda $^{2}$, \\ Yoshihiro Takaki ${ }^{2} \&$ Shigeru Deguchi ${ }^{1, *}$
}

\section{Research Center for Bioscience and Nanoscience, Japan Agency for Marine-Earth}

Science and Technology (JAMSTEC), 2-15 Natsushima-cho, Yokosuka 237-0061, Japan

2. SUGAR Program, JAMSTEC, 2-15 Natsushima-cho, Yokosuka 237-0061, Japan

Correspondence should be addressed to S.D. (shigeru.deguchi@jamstec.go.jp).

†. Present address. Bioengineering Lab. Co., Ltd., 657 Nagatake, Midori-ku, Sagamihara 252-0154, Japan

\begin{abstract}
Substrates for enzymatic reactions are often insoluble in water. Typical examples include structural polysaccharides such as cellulose and chitin. Being the most abundant and the second most abundant biomass, respectively, their enzymatic degradation not only plays a dominant role in the global carbon cycle but also is of tremendous technological importance for providing primary feedstocks for bio-based chemicals. Plastics have become very important in this category in recent years as a keen interest in the bioremediation of plastic pollution has emerged. Despite the significance, however, assaying enzymatic degradation of the water-insoluble substrate has remained technically challenging, primarily because only the surface of the substrate is accessible to the enzymes and the reaction proceeds very slowly compared with those of water-soluble substrates. Here we show an ultra-sensitive and semiquantitative assay for enzymatic hydrolysis of cellulose. By combining nanofibrous matrices with piezo-driven inkjet printing and optical profilometry, enzymatic
\end{abstract}


hydrolysis of less than 1 nanogram of crystalline cellulose was successfully quantified. Unprecedented genetic diversity of cellulase was revealed when the same principle was applied for elucidating microbial degradation of cellulose in the deep sea. This work demonstrates that truly interdisciplinary efforts, encompassing diverse disciplines from nanotechnology to microbiology, are crucial to address scientific and technological problems towards sustainability. 


\section{Introduction}

Cellulose, which is a linear and nonionic polysaccharide made of glucose connected via $\beta-1-4$ glycosidic linkages, ${ }^{1}$ is the major component of plant cell walls and is the most abundant organic polymer with an estimated annual production of $\sim 100$ billion dry tons. ${ }^{2}$ Hydrolysis of cellulose by microbial cellulases is a primary process for turnover of photosynthetically fixed carbon in the global carbon cycle..$^{2-4}$ Microbial cellulases are also deemed crucial components to establish biorefinery from this inexhaustible feedstock. ${ }^{2,5,6}$

All cellulases cleave a $\beta-1-4$ glycosidic bond in water-soluble substrates such as cellodextrins and carboxymethyl cellulose (CMC), and water-insoluble amorphous cellulose. ${ }^{7}$ However, crystalline cellulose is resistant to enzymatic hydrolysis due to extensive and highly ordered hydrogen bonding networks between the cellulose chains, and its hydrolysis usually requires synergetic actions of multiple enzymes. Glycoside hydrolases (GHs) that are classified as endoglucanase and exoglucanase (or cellobiohydrolase) are playing a major role in generating short oligosaccharides. ${ }^{6,8}$ Especially, exoglucanases are known to be capable of breaking down crystalline regions of cellulose by detaching single cellulose chains from crystal surface and guiding them into their long tunnel-shaped catalytic sites for hydrolysis. ${ }^{9}$ The efficient hydrolysis is achieved with the assistance of oxidative enzymes such as copper-dependent lytic polysaccharide monooxygenases which cleave internal glycosidic bond of crystalline cellulose and make it accessible for GHs. ${ }^{10}$ Some microorganisms produce these enzymes as free enzymes which are often modular enzymes connected with carbohydrate binding domains (CBMs), while some anaerobic microorganisms produce them as elaborate supramolecular complexes called cellulosomes. ${ }^{4,6,11}$ 
Like most enzymatic reactions, quantitative assay of enzymatic hydrolysis of cellulose was usually carried out by measuring accumulation of end-products. ${ }^{12}$ However, existing methods were insensitive and time-consuming, primarily due to recalcitrance and water-insolubility of crystalline cellulose. ${ }^{2,12}$ These difficulties facilitated introduction of unconventional techniques to study cellulase reaction. For example, high-speed atomic force microscopy ${ }^{13}$ was successfully used to visualize cellulose hydrolysis by individual enzyme molecules and revealed that the reaction was affected by congestion of the cellulase molecules on the cellulose surface. ${ }^{14,15} \mathrm{We}$ used nanofibrous substrates, combined with ink-jet patterning and 3D laser profilometry, to develop an ultra-sensitive and quantitative assay for enzymatic hydrolysis of crystalline cellulose, which we call SPOT (Surface Pitting Observation Technology).

\section{Results}

Surface pitting on nanofibrous cellulose matrix. When crystalline cellulose (Figure 1a, cellulose-I, 58.2\% crystallinity) ${ }^{16}$ and nanofibrous cellulose (Figure 1b, cellulose-II, $44.5 \%$ crystallinity) ${ }^{16}$ were subjected to enzymatic hydrolysis using fungal cellulases from Trichoderma viride, ${ }^{17}$ the amount of glucose that was produced from nanofibrous cellulose after $30 \mathrm{~min}$ of reaction $(1.4 \mathrm{mg} / \mathrm{mL})$ was 3.5 times higher than that from the cellulose powder $(0.4 \mathrm{mg} / \mathrm{mL})$ (Figure $\mathrm{S} 1 \mathrm{a})$. The difference would be ascribed to a significantly larger specific surface area of nanofibrous cellulose (220 $\mathrm{m}^{2} / \mathrm{g}$ ) than that of cellulose powder $\left(1.8 \mathrm{~m}^{2} / \mathrm{g}\right.$ ) (Figure $\mathrm{S} 1 \mathrm{~b}$ ), as well as the difference in the crystalline form and the crystallinity. ${ }^{16}$

During the reaction, initially turbid dispersion containing fine particles made of nanofibrous cellulose was cleared in $2 \mathrm{~h}$, whereas the dispersion containing the cellulose powder remained turbid even after $4 \mathrm{~h}$ of hydrolysis (Figures $1 \mathrm{c}$ and $1 \mathrm{~d}$, Movie S1). The 
observation shows that hydrolysis of water-insoluble substrates such as cellulose can alternatively be assayed by following the loss of the substrates.

In SPOT, this was realized by performing the reaction on the surface of nanofibrous cellulose matrices. When 18,36 , and $54 \mathrm{pL}$ of a solution containing the cellulases $(10 \mathrm{mg} / \mathrm{mL})$ were inkjet-patterned on the flat surface of a wet porous matrix made of nanofibrous cellulose,${ }^{16}$ the enzymes hydrolyzed the nanofibers and destroyed their three-dimensional network, leading to formation of pits on the surface (Figure 1e). Actual mass of the cellulases that were deposited was $0.18,0.36$, and $0.54 \mathrm{ng}$, respectively.

We used 3D profilometry to obtain a height map of the pitted surface in a nondestructive manner (Figure 1f). The pit formation was reproducible, because crosssectional profiles that were obtained from the map along the white lines superimposed on each other very well (Figure $1 \mathrm{~g}$ ). It is also evident that the pits became deeper with increasing the amount of the deposited cellulases. These results suggest the surface pitting can potentially be used as a quantitative indicator to follow cellulose hydrolysis using an extremely small amount of enzyme.

Quantifying surface pitting to assay cellulase reaction. Measurements of pit formation kinetics revealed that the surface pitting was not only a quantitative indicator but also an ultra-sensitive indicator to assay cellulase reaction. The pit grew after initiating the reaction by cellulase deposition and became deeper as a function of reaction time (Figure $2 \mathrm{a}$ and $2 \mathrm{~b}$ ). The cross-sectional profile has been commonly used to analyze three-dimensional topographic features (Figure 2c), but it does not give information that is necessary to follow the progress of the reaction such as mass of hydrolyzed cellulose. Instead, we measured the volume of the pit (Figure $2 \mathrm{~d}$ ) and 
calculated actual mass of hydrolyzed cellulose (illustrated by thick white lines in Figure $2 \mathrm{~d})$ by multiplying it with the fiber density of the matrix $\left(0.03 \mathrm{~g} / \mathrm{cm}^{3}\right)$.

Measured mass of hydrolyzed cellulose increased linearly with the reaction time up to $15 \mathrm{~min}$, but the growth slowed down thereafter (Figure 2e). Initial hydrolysis rates, which were obtained from the slope of linear fits to the data points within $15 \mathrm{~min}$, were between $60-180 \mathrm{pg} \cdot \mathrm{min}^{-1}$ and proportional to the amount of the deposited cellulases (Figure 2f). It should be noted that the actual amount of hydrolyzed cellulose might have been much smaller, as it was sufficient to hydrolyze only parts of nanofibers to separate them from the rest for destroying the porous framework and pitting the surface.

\section{Surface pitting as selective indicator for hydrolysis of crystalline cellulose.}

With crystallinity index of $0.45,{ }^{16}$ nanofibrous cellulose is a blend of recalcitrant crystalline domains and easily hydrolyzable amorphous domains. Hydrolysis of the latter alone may also lead to pit formation. However, we found that a $\beta-1-4$ endoglucanase from Bacillus agaradhaerens, which hydrolyses CMC and amorphous cellulose but not crystalline cellulose,${ }^{18}$ showed near-zero hydrolytic activity against nanofibrous cellulose (Table S1), suggesting that surface pitting is a selective indicator for hydrolysis of crystalline cellulose.

\section{Surface pitting for recognizing microbial production of cellulase. Surface}

pitting can also be used to recognize production of cellulases by microorganisms with high sensitivity. It was previously reported that various microorganisms (e.g., Escherichia coli, Bacillus subtilis, Saccharomyces cerevisiae, and several extremophiles) formed colonies when they were cultured on the surface of nanofibrous cellulose plates containing appropriate nutrients (Figure 3a). ${ }^{16,19}$ When a model cellulase-producer, Saccharophagus degradans $2-40^{\mathrm{T}}$ (ATCC 43961), ${ }^{20,21}$ was cultured, 
however, cellulases that the organism produced hydrolyzed nanofibrous cellulose and pit the plate surface (Figures 3b-e). A small and shallow pit appeared after incubation for $24 \mathrm{~h}$ and grew in size with the incubation time (Figure 3e).

There were three notable differences between enzymatic and microbial surface pitting. First, the growth of the enzymatic pit occurred primarily in the depth direction (Figure $2 b$ ), whereas the microbial pit grew both in the depth and width directions (Figure 3e). Analysis of the cross-sectional profiles confirmed that the microbial pit grew to both directions at similar rates (Figure 3f). The difference in the pit growth behavior could be ascribed to the way cellulase molecules were supplied to the reaction. The cellulase molecules were deposited all at once by inkjet-patterning in enzymatic pitting. In microbial pitting, however, the enzyme molecules were continuously released from growing cells that were attached to the surface of the pit.

Second, the size of the pit the bacterium produced (Figure 3g) was an order of magnitude smaller than those obtained by depositing a solution of the cellulases (Figure 2e). In the case of the pit formed after incubation for $24 \mathrm{~h}$ (Figure $3 \mathrm{~b}$ ), the amount of hydrolyzed cellulose was $1.6 \mathrm{pg}$, which corresponds to the weight of one hundredth of a grain of cellulose (Figure S4). Because the specific gravity of cellulose is $1.48-1.63$ $\mathrm{g} \cdot \mathrm{cm}^{-3},{ }^{22}$ the volume of 1.6 picograms of monolithic cellulose is $\sim 1 \times 10^{-12} \mathrm{~cm}^{3}$, which is too small to be detected by the present experimental set-up. Clearly, the low fiber density of the porous substrate $\left(0.03 \mathrm{~g}^{\bullet} \mathrm{cm}^{-3}\right)$ facilitated detection of cellulose hydrolysis because hydrolysis of a minute amount of cellulose led to a large volume change (Figure 2d).

Third, the microbial pitting did not slow down but rather accelerated at longer incubation times (Figure 3g). Further incubation of $S$. degradans for 1 week led to formation of millimeter-sized macroscopic pits (Figure S5), thereby allowing facile 
recognition of microbial production of cellulases by visual inspection (Figure S6). Moreover, compared with a standard method for recognizing microbial cellulolytic activity toward crystalline cellulose ${ }^{23}$ activity recognition by surface pitting took significantly shorter time (Figures S7). Surface pitting was also a selective indicator for microbial production of cellulases that hydrolyze crystalline cellulose (Figure S8).

Screening of cellulolytic bacteria from the deep sea. Culture-independent metagenomic approaches have been successfully used to mine cellulase genes and elucidate diversity of microbial cellulases..$^{24,25}$ For obtaining novel cellulases from natural environments, however, the metagenomic approach should be complemented by conventional culture-dependent methods. ${ }^{25}$ Culture-dependent screening of cellulolytic microorganisms takes full advantage of the ultra-sensitivity of surface pitting for identifying cellulase activity. In selecting cellulolytic microorganisms out of an overwhelming majority of non-cellulolytic ones in environmental samples, ${ }^{3}$ ultrasensitive detection is crucial to recognize microbial production of low concentrations of cellulases.

To demonstrate the performance, we deployed sterilized nanofibrous cellulose plates in the deep sea using research submersibles (Figure S9), and studied cellulolytic activity of deep-sea bacteria that attached to the plate after recovery. From pits that were formed on the plate surface (Figure 3h), we have successfully isolated six cellulolytic strains. SEM image in Figure 3i shows one of the isolates, strain GE09, grew and pit the surface of a nanofibrous cellulose plate. The surface of the pit was covered with a dense mat of rod-shaped cells. Identification using $16 \mathrm{~S}$ rRNA gene sequences ${ }^{26}$ showed that four of the isolates were likely classified as new bacterial species or genera (Figure S10). We did not detect cellulolytic activity of bacteria in a parallel screening attempt using a conventional method (agar plates with crystalline cellulose powder), suggesting 
that surface pitting on nanofibrous cellulose allowed isolating novel cellulolytic microorganisms that the conventional method failed to recognize (Figure S9).

Insights into plant cell wall degradation by a deep-sea bacterium GE09. We determined the complete genome of one of the isolated bacterial species, strain GE09, as the statistical genome features were shown in Table S2. A large number of extracellular polysaccharide-degrading enzymes were identified in its genome and it was probable that they were playing important roles to acquire nutrients and fixed carbon. Seventy-nine CDSs were predicted to be targeted to secretion and code at least one domain sequence assigned to the families in the carbohydrate-active enzymes database (CAZy database, http://www.cazy.org). ${ }^{27}$ Figure 4a shows its classification based on their target substrates in comparison to closely related marine $\gamma$ proteobacteria T. turnerae ${ }^{28}$ and S. degradans. ${ }^{29}$ Predicted enzymes targeting cellulose, hemicellulose (xylan and mannan) and pectin, which are the major components of plant cell walls, accounted a remarkable proportion of secreted CAZymes in GE09 (Figure 4a, left). In contrast, there were only a few candidate genes of enzymes that dedicated to marine polysaccharide degradation such as alginate and laminarin. Chitinase and agarase genes were not found in their genomes, which agrees with our experimental observation that they could not grow on chitin and agar as sole carbon sources. These results indicate that GE09 specializes to degrade plant cell walls in analogy with an endosymbiont of shipworm, T. turnerae, rather than diversifies into degradation of marine polysaccharides as free-living bacterium $S$. degradans does.

Characteristics of cellulases from the deep-sea bacterium GE09. The strain GE09 encoded more than 10 genes of major cellulases in their genomes, whose catalytic domains were classified into glycoside hydrolase (GH) family 5, 6 and 9 in the CAZy database as listed in Table S3. Many of them were predicted to have the type II secretion signal, which indicated that proteins were anchored to bacterial cellular 
membranes rather than secreted as free-enzymes. The existence of several exo-type cellulases containing GH family 6 domains was noteworthy as they were necessary for degrading the crystalline part of cellulose. All genes of GH family 6 domain were classified into branches that were distinctly different from the majority of bacterial CBH genes in phylogenetic analysis (Figure $4 b$, branches indicated as a and b). As is shown in one of the modeled structures of GH family 6 domains of GE09 in Figure 4b (right, top panel), there were many sequence insertions (blue-colored regions) in the GE09 cellulase gene relative to the experimentally determined structure of bacterial $\mathrm{CBH}$, Thermobifida fusca Cel6B (Figure 4b right, bottom panel). Although its core structure and loops adjacent to the active sites were structurally conserved, the inserted loop structures were not modeled well. The regions proximity to the ligand binding site would especially affect its catalytic activity as they had been known to be closely related to the substrate uptake and processive degradation of insoluble crystalline substrates. More covered active sites have been known to make enzymes tighter binding to substrates. ${ }^{30,31}$ Two genes from GE09 shown in branch $b$ in the phylogenetic tree were unsure to be catalytically active as they lacked essential catalytic residues.

Another characteristic feature of the cellulase genes from the deep-sea bacterium was their unique molecular architectures (Figure 4c). Instead of carbohydrate binding modules (CBMs) that are usually found in combination with catalytic domains of bacterial cellulases, the most commonly found domain was polycystic kidney disease (PKD) in GH family 6 cellulase genes and there were no CBMs that can be judged only by genome sequencing results. In addition, there was an unknown sequence composed of $\sim 250$ amino acids at the C-terminus of a putative cellulase gene MERGE09_P3218 (Figure 4c, upper), which commonly used databases such as BLAST, Pfam and dbCAN failed to annotate. We found that it had structural similarity to the cellulose induced protein I (CIP1), which was known as a protein of unknown function that were coregulated with cellulases. ${ }^{32}$ There were completely unfamiliar modular architectures 
such as the sequence MERGE09_P1318 (Figure4c, bottom) and the sequence MERGE09_P1043 that had peculiar characteristics in terms of its high-molecular weight $(\sim 3,400$ amino acids, Table S3). These findings suggest that some remarkable changes were occurred in the choices and combinations of accessory domains, not only in the sequences of catalytic core domains.

\section{Discussion}

Mass loss of thin films of nanofibrous cellulose was measured by a quartz crystal microbalance with dissipation monitoring (QCM-D) to study kinetics of their hydrolysis. ${ }^{33}$ The method allowed real-time observation of enzyme binding and hydrolysis kinetics, and also gave information about morphological changes of the substrates. Our results complement the previous study by demonstrating that the volume loss can also be used for the analysis. Unlike QCM-D measurements, in which the mass loss is registered by a change in resonant frequency of a quartz crystal, the actual weight of hydrolyzed cellulose can be estimated by SPOT. Furthermore, SPOT significantly extend the substrate-loss based analysis by enabling a new realm of applications such as analysis using pL-quantities of a cellulase solution, detection of microbial production of cellulases that hydrolyze crystalline cellulose, and screening of cellulolytic microorganisms. SPOT could also be used to develop massively parallel analysis (Figures 1e and 1f).

Microorganisms are central to carbon cycling in marine environments, even at the deepest point of the ocean. ${ }^{34}$ Yet, the deep sea is a highly unconventional place to look for cellulolytic bacteria because sunlight does not reach below $200 \mathrm{~m}$ and cellulose is not produced in situ. ${ }^{35}$ This does not imply the deep sea is completely deprived of cellulose, however, because the vertical transport of cellulose from the surface photosynthetic world occurs. ${ }^{36}$ Cellulolytic bacteria may play a key ecological role in 
unidentified carbon cycle and the food web also in the deep ocean, ${ }^{37}$ but such a bacterium was not known.

Successful isolation of novel cellulolytic bacteria from such environments not only demonstrates the advantage of surface pitting for detecting microbial cellulase production, but also extends the promises of marine microbial resources for cellulosic biotechnology to deep-sea microorganisms. ${ }^{38,39}$ Considering the fact that the majority of microorganisms in any given environments have not been cultured and characterized, ${ }^{40}$ it is likely that application of SPOT to conventional terrestrial sources would likely lead to discoveries of previously unknown cellulose degraders. It can also be used to screen for thermophilic cellulolytic microorganisms, ${ }^{16,19}$ whose cellulases have various advantages for industrial applications. ${ }^{41,42}$

Predictions of cellulolytic enzymes from the isolated bacterium GE09 resulted in finding of unique cellulases from deep-sea. The preferential repertoire of plant cell wall degrading enzymes suggests their substantial role in degradation of cellulose in the oligotrophic soil at the dark ocean floor. Their strategy of cellulose degradation would be different from well-studied free enzyme cocktail or cellulosome style degradation, as they utilize cell-membrane associated cellulases with unknown domain structures. The isolated deep-sea bacteria were unable to glow in usual cultivation conditions such as agar plates and were previously hard to find because there was no way to detect cellulose-degradation activity of microorganisms whose cultivation conditions are not established. The high-sensitive technology, SPOT, enabled to open an opportunity to detect undiscovered cellulose-degrading microorganisms to find new genes and enzymes.

In the late 19th century, nanofibrous matrices of gelatin and agar were used for developing solidified media, in which culture media were confined in pores of the 
matrices. The solidified media allowed biologists to isolate pure cultures of microorganisms and helped them to reveal that several diseases were caused by microorganisms. ${ }^{43}$ The finding lead to the establishment of modern microbiology, which had a tremendous impact on biology at that time along with the Darwinian theory of evolution. ${ }^{44}$ Unfortunately, the role that the polymeric nanomaterials played over a century ago for advancing the knowledge on microorganisms is not well recognized in nanotechnology today despite the fact that their application in medical biotechnology (i.e., "red" biotechnology) is actively pursued. The present study reinstates the promise of nanomaterials toward microbial biotechnology (i.e., "white" biotechnology), ${ }^{45,46}$ which may be called white nanobiotechnology.

Acknowledgments. Yoshihiro Fujiwara, Satoshi Konishi, Sumihiro Koyama, Kaoru Kubokawa, Tetsuya Miwa, Masayuki Miyazaki, and Yuichi Nogi assisted with the collection of the samples. We thank Tohru Hamatani for kindly supplying us with Meicelase, Yasushi Hori for assistance in inkjet patterning, Katsuyuki Uematsu and Akihiro Tame for technical assistance in SEM observations, and Ryusuke Nabata for measurements of the specific surface area. We are grateful to Koki Horikoshi, Susumu Ito, Kaoru Tsujii, Tohru Kobayashi, Kiyohiko Igarashi and Taku Uchiyama for stimulating discussion. Parts of this work were financially supported by Grant-in-Aid for Scientific Research on Innovative Areas (Grant Number 25120512, to S.D., M. Tsudome), Grant-in-Aid for Young Scientists (B) (Grant Number 25850127, to M. Tsudome; Grant Number 26850226, to K.U.), and Grant-in-Aid for Young Scientists (Grant Number 19K15956, to M. Tachioka) from Japan Society for the Promotion of Science, the Shimadzu Science Foundation (to S.D.), the Japan Prize Foundation (to M. Tsudome), and Kyokuto Pharmaceutical Industrial Co., Ltd. (to S.D., M. Tsudome, and K.U.). Kyokuto Pharmaceutical Industrial Co., Ltd. is also acknowledged for placing the 3D laser profiler at our disposal.

\section{Author Contributions.}

Mikiko Tsudome: Methodology, Investigation, Visualization, Funding acquisition 
Mikako Tachioka: Methodology, Investigation, Formal analysis, Writing - Original Draft, Visualization, Funding acquisition

Kohsuke Uchimura: Methodology, Investigation, Funding acquisition

Miwako Tsuda: Methodology, Investigation

Yoshihiro Takaki: Formal analysis, Writing - Original Draft

Shigeru Deguchi: Conceptualization, Investigation, Formal analysis, Writing - Original Draft, Writing Review \& Editing, Visualization, Supervision, Project administration, Funding acquisition

Competing financial interests. JAMSTEC has patents relating to production and uses of the nanofibrous cellulose plate, of which S.D. and M. Tsudome are named inventors. S.D. and M. Tsudome have received research support and may profit from the sale of the nanofibrous cellulose plate, which has been developed by Kyokuto Pharmaceutical Industrial Co., Ltd., Japan, through a joint research agreement with JAMSTEC. M. Tachioka, K.U., M. Tsuda and Y.T. declare no competing financial interest. 


\section{Online Methods}

Preparation of nanofibrous cellulose. Nanofibrous cellulose plates were prepared according to the literature. ${ }^{16,19}$ Briefly, cellulose (microcrystalline cellulose for thinlayer chromatography, Merck, Darmstadt, Germany) was dissolved in a hot saturated solution of $\mathrm{Ca}(\mathrm{SCN})_{2}$ in water at the concentration of 0.03 or $0.01 \mathrm{~g}^{\bullet} \mathrm{cm}^{-3}$. The solution was allowed to solidify at room temperature overnight, during which the porous network of nanofibrous crystalline cellulose was spontaneously formed. $\mathrm{Ca}(\mathrm{SCN})_{2}$ was removed from the solidified plates by washing them with methanol and water.

Enzymatic surface pitting. A solution containing $10 \mathrm{mg} / \mathrm{mL}$ of cellulases from $T$. viride (Meicelase, Meiji Seika, Tokyo, Japan) was deposited onto a flat surface of nanofibrous cellulose plate containing $0.1 \mathrm{M}$ acetate buffer ( $\mathrm{pH} 4.8)$. An inkjet patterning device (LaboJet-500Bio, Microjet Corporation, Shiojiri, Japan) equipped with an IJHBS-10 inkjet head was used for deposition. The instrument was adjusted to deposit $9 \mathrm{pL}$ of the cellulase solution at one shot. The amount of a deposited solution was controlled by repeatedly depositing the solution in a same place. Reactions were allowed to proceed at room temperature.

Laser profilometry. Pits on the surface of nanofibrous cellulose matrices were quantified using a VK-9700 Generation II (Keyence, Osaka, Japan), which is a confocal laser scanning microscope working in reflection mode. The surface of an object was laterally scanned using a laser $(\lambda=408 \mathrm{~nm})$, and the intensity of reflected light from the surface, $I$, was measured by a photomultiplier. A field of view was divided to $1024 \times$ 768 pixels. Measurements were repeated while scanning the object in the depth $(z)$ direction to obtain a stack of images, of which each pixel was associated with $z$ and $I$. A focal position of a pixel was given at $z$ where the strongest $I$ was recorded. A 3D height map was generated by locating focal positions of all pixels in an image. Pit volume was 
determined from the 3D height map using Scanning Probe Image Processor (SPIP, Image Metrology, Hørsholm, Denmark).

Surface pitting by $\boldsymbol{S}$. degradans. S. degradans $2-40^{\mathrm{T}}$ (ATCC 43961) was cultured in marine broth (Difco 2216) supplemented with cellobiose $(0.1 \mathrm{wt} \%)$ at $30^{\circ} \mathrm{C}$ for 3 days. The culture was diluted $10^{6}$ times with artificial seawater (ASW, Marine Art SF-1, Senju Pharmaceutical, Osaka, Japan), spread on nanofibrous cellulose plates containing ASW plus ammonium sulfate $(1 \mathrm{mM})$ and trace minerals (Solution A from Teredinibacter medium, ATCC medium no. 1983), and incubated at $30^{\circ} \mathrm{C}$.

Screening of deep-sea cellulase producers. Nanofibrous cellulose plates containing pure water were autoclaved at $121^{\circ} \mathrm{C}$ for $20 \mathrm{~min}$. The plates were then transferred to a polystyrene Petri dish, and the lid and body were glued. A small gap was left between them so that water could go in and out of the dish, whereas the cellulose plate could not escape from the dish. Sampling operations were performed by exposing nanofibrous cellulose to deep-sea water (at the depth between 141 and $654 \mathrm{~m}$ ) using the unmanned remotely operate vehicle Hyper-Dolphin or the manned submersible Shinkai 6500. Detailed sampling conditions are described in Supporting Information. The recovered plates were crushed into fine particles and dispersed in ASW. The dispersions were spread on fresh cellulose plates containing low nutrient media. The plates were incubated at $15^{\circ}$ or $20^{\circ} \mathrm{C}$, and the plate surface was inspected visually for the formation of pits. When pits were formed on the surface of cellulose plates, microorganisms in the pits were picked up with toothpicks, dispersed in ASW, and then spread on fresh cellulose plates.

Characterisation of cellulases from a deep-sea bacterium. One of the isolated cellulolytic strain GE09 was grown in liquid culture and the whole genome were sequenced by using Illumina MiSeq platform. CAZyme genes were identified using dbCAN2 ${ }^{47}$ and subcellular localization was predicted using LipoP. ${ }^{48}$ Substrate 
specificities were predicted according to CAZy family classification and completed by manual annotation. The genes containing GH family 6 domains were further annotated by reference to Pfam database, BLAST searches and structural modeling using SWISSMODEL. ${ }^{49}$ The phylogenetic tree of GH family 6 domains was constructed using MAFFT with E-NS-2 method. ${ }^{50}$ 


\section{References}

1. Klemm, D., Heublein, B., Fink, H. P. \& Bohn, A. Cellulose: Fascinating biopolymer and sustainable raw material. Angew. Chem. Int. Ed. 44, 3358-3393 (2005).

2. Zhang, Y.-H. P., Himmel, M. E. \& Mielenz, J. R. Outlook for cellulase improvement: Screening and selection strategies. Biotechnol. Adv. 24, 452-481 (2006).

3. Cragg, S. M. et al. Lignocellulose degradation mechanisms across the Tree of Life. Curr. Opin. Chem. Biol. 29, 108-119 (2015).

4. Lynd, L. R., Weimer, P. J., Zyl, W. H. van \& Pretorius, I. S. Microbial cellulose utilization: fundamentals and biotechnology. Microbiol. Mol. Biol. Rev. 66, 506-577 (2002).

5. Menon, V. \& Rao, M. Trends in bioconversion of lignocellulose: Biofuels, platform chemicals \& biorefinery concept. Prog. Energy Combust. Sci. 38, 522-550 (2012).

6. Payne, C. M. et al. Fungal cellulases. Chem. Rev. 115, 1308-1448 (2015).

7. Bayer, E. A., Chanzy, H., Lamed, R. \& Shoham, Y. Cellulose, cellulases and cellulosomes. Curr. Opin. Struct. Biol. 8, 548-557 (1998).

8. Hemsworth, G. R., Déjean, G., Davies, G. J. \& Brumer, H. Learning from microbial strategies for polysaccharide degradation. Biochem. Soc. Trans. 44, 94-108 (2016).

9. Beckham, G. T. et al. Towards a molecular-level theory of carbohydrate processivity in glycoside hydrolases. Curr. Opin. Biotechnol. 27, 96-106 (2014).

10. Beeson, W. T., Vu, V. V., Span, E. A., Phillips, C. M. \& Marletta, M. A. Cellulose degradation by polysaccharide monooxygenases. Annu. Rev. Biochem. 84, 923-946 (2015).

11. Fontes, C. M. G. A. \& Gilbert, H. J. Cellulosomes: Highly efficient nanomachines designed to deconstruct plant cell wall complex carbohydrates. Annu. Rev. Biochem. 79, 655-681 (2010).

12. Dashtban, M., Maki, M., Leung, K. T., Mao, C. \& Qin, W. Cellulase activities in biomass conversion: measurement methods and comparison. Crit. Rev. Biotechnol. 30, 302-309 (2010).

13. Ando, T. High-speed atomic force microscopy coming of age. Nanotechnology 23, 062001 (2012).

14. Igarashi, K. et al. Traffic jams reduce hydrolytic efficiency of cellulase on cellulose surface. Science 333, 1279-1282 (2011).

15. Igarashi, K. Cooperative biomass breakdown. Nat. Chem. Biol. 9, 350-351 (2013). 
16. Deguchi, S. et al. Preparation and characterisation of nanofibrous cellulose plate as a new solid support for microbial culture. Soft Matter 3, 1170-1175 (2007).

17. Winarni, I. et al. Improvement of enzymatic saccharification of unbleached cedar pulp with amphipathic lignin derivatives. BioResources 8, 2195-2208 (2013).

18. Hirasawa, K. et al. Salt-activated endoglucanase of a strain of alkaliphilic Bacillus agaradhaerens. Antonie van Leeuwenhoek 89, 211-219 (2006).

19. Tsudome, M., Deguchi, S., Tsujii, K., Ito, S. \& Horikoshi, K. Versatile solidified nanofibrous cellulose-containing media for growth of extremophiles. Appl. Environ. Microbiol. 75, 4616-4619 (2009).

20. Taylor, L. E. et al. Complete cellulase system in the marine bacterium Saccharophagus degradans strain 2-40 ${ }^{\mathrm{T}}$. J. Bacteriol. 188, 3849-3861 (2006).

21. Jung, Y. H. et al. Feasibility test of utilizing Saccharophagus degradans $2-40^{\mathrm{T}}$ as the source of crude enzyme for the saccharification of lignocellulose. Bioprocess Biosyst. Eng. 1-4 (2013) doi:10.1007/s00449-013-1040-1.

22. Aulin, C., Gällstedt, M. \& Lindström, T. Oxygen and oil barrier properties of microfibrillated cellulose films and coatings. Cellulose 17, 559-574 (2010).

23. Hendricks, C. W., Doyle, J. D. \& Hugley, B. A new solid medium for enumerating cellulose-utilizing bacteria in soil. Appl. Environ. Microbiol. 61, 2016-2019 (1995).

24. Li, L.-L., McCorkle, S. R., Monchy, S., Taghavi, S. \& Lelie, D. van der.

Bioprospecting metagenomes: glycosyl hydrolases for converting biomass. Biotechnol. Biofuels 2, 1-11 (2009).

25. Duan, C.-J. \& Feng, J.-X. Mining metagenomes for novel cellulase genes. Biotechnol. Lett. 32, 1765-1775 (2010).

26. Weisburg, W. G., Barns, S. M., Pelletier, D. A. \& Lane, D. J. 16S ribosomal DNA amplification for phylogenetic study. J. Bacteriol. 173, 697-703 (1991).

27. Lombard, V., Ramulu, H. G., Drula, E., Coutinho, P. M. \& Henrissat, B. The carbohydrate-active enzymes database (CAZy) in 2013. Nucleic Acids Res. 42, D490D495 (2013).

28. Yang, J. C. et al. The complete genome of Teredinibacter turnerae T7901: An intracellular endosymbiont of marine wood-boring bivalves (shipworms). PLoS ONE 4, e6085 EP- (2009).

29. Weiner, R. M. et al. Complete genome sequence of the complex carbohydratedegrading marine bacterium, Saccharophagus degradans strain $2-40^{\mathrm{T}}$. PLoS Genet. 4, e1000087 EP- (2008).

30. Horn, S. J. et al. Costs and benefits of processivity in enzymatic degradation of recalcitrant polysaccharides. Proc. Natl. Acad. Sci. U.S.A. 103, 18089-18094 (2006). 
31. Nakamura, A. et al. Trade-off between processivity and hydrolytic velocity of cellobiohydrolases at the surface of crystalline cellulose. J. Am. Chem. Soc. 136, 45844592 (2014).

32. Jacobson, F. et al. The crystal structure of the core domain of a cellulose induced protein (Cip1) from Hypocrea jecorina, at $1.5 \AA$ Å resolution. PLoS ONE 8, e 70562 (2013).

33. Ahola, S., Turon, X., Österberg, M., Laine, J. \& Rojas, O. J. Enzymatic hydrolysis of native cellulose nanofibrils and other cellulose model films: Effect of surface structure. Langmuir 24, 11592-11599 (2008).

34. Glud, R. N. et al. High rates of microbial carbon turnover in sediments in the deepest oceanic trench on Earth. Nature Geoscience 6, 284-288 (2013).

35. Littler, M. M., Littler, D. S., Blair, S. M. \& Norris, J. N. Deepest known plant life discovered on an uncharted seamount. Science 227, 57-59 (1985).

36. Alldredge, A. L., Passow, U. \& Haddock, H. D. The characteristics and transparent exopolymer particle (TEP) content of marine snow formed from thecate dinoflagellates. J. Plankton Res. 20, 393-406 (1998).

37. D'Hondt, S. et al. Distributions of microbial activities in deep subseafloor sediments. Science 306, 2216-2221 (2004).

38. Kennedy, J., Marchesi, J. R. \& Dobson, A. D. Marine metagenomics: strategies for the discovery of novel enzymes with biotechnological applications from marine environments. Microb. Cell Fact. 7, 1-8 (2008).

39. Intriago, P. Marine Microorganisms: perspectives for getting involved in cellulosic ethanol. AMB Express 2, 1-12 (2012).

40. Zengler, K. Central role of the cell in microbial ecology. Microbiol. Mol. Biol. Rev. 73, 712-729 (2009).

41. Maki, M., Leung, K. T. \& Qin, W. The prospects of cellulase-producing bacteria for the bioconversion of lignocellulosic biomass. Int. J. Biol. Sci. 5, 500-516 (2009).

42. Graham, J. E. et al. Identification and characterization of a multidomain hyperthermophilic cellulase from an archaeal enrichment. Nat. Commun. 2, 1-9 (2011).

43. Hashsham, S. A. Culture Techniques. in (eds. Reddy, C. A. et al.) 270-285 (ASM Press, 2007).

44. Twenty Years. Nature 41, 1-5 (1889).

45. Frazzetto, G. White biotechnology. EMBO Rep. 4, 835-837 (2003).

46. Bevan, M. W. \& Franssen, M. C. R. Investing in green and white biotech. Nat. Biotechnol. 24, 765-767 (2006).

47. Zhang, H. et al. dbCAN2: a meta server for automated carbohydrate-active enzyme annotation. Nucleic Acids Res. 46, W95-W101 (2018). 
48. Juncker, A. S. et al. Prediction of lipoprotein signal peptides in Gram-negative bacteria. Protein Sci. 12, 1652-1662 (2003).

49. Waterhouse, A. et al. SWISS-MODEL: homology modelling of protein structures and complexes. Nucleic Acids Res. 46, W296-W303 (2018).

50. Katoh, K., Rozewicki, J. \& Yamada, K. D. MAFFT online service: multiple sequence alignment, interactive sequence choice and visualization. Brief. Bioinformatics 20, 1160-1166 (2019). 


\section{Figure legends}

Figure 1 | Surface pitting on nanofibrous cellulose matrix. (a, b) SEM images comparing morphology of (a) untreated cellulose powder and (b) nanofibrous cellulose (taken from ref. ${ }^{16}$ ). (c, d) Photographs showing nanofibrous cellulose (left) and untreated cellulose powder (right) after enzymatic digestion for (c) 0 min and (d) 120 min in $0.1 \mathrm{M}$ acetate buffer (pH $4.8)$ at $40^{\circ} \mathrm{C}$. A video of the hydrolysis process is available as supplementary information (Movie S1). (e and f) Omnifocal microscopic image (e) and 3D height map (f) of pits formed on the surface of a nanofibrous cellulose $(0.03$ $\mathrm{g} \cdot \mathrm{cm}^{-3}$ cellulose) containing $0.1 \mathrm{M}$ acetate buffer $(\mathrm{pH} 4.8)$ after depositing 0.18 , 0.36 , and $0.54 \mathrm{ng}$ of the cellulases (shown by the numbers in the figure). Scale bar in e represents $500 \mu \mathrm{m}$. Vertical lines in e are artifacts that were introduced when multiple images were computationally stitched to generate a panorama image. (g) A cross-sectional profile of the pits, which were measured along the white lines in $\mathbf{f}$. Profiles were horizontally shifted arbitrarily to match positions of the peaks.

Figure 2 | Quantifying pitting kinetics on nanofibrous cellulose matrix. (a) 3D topographic images of a pit formed on the surface of a nanofibrous cellulose matrix $\left(0.03 \mathrm{~g} \cdot \mathrm{cm}^{-3}\right.$ cellulose) containing $0.1 \mathrm{M}$ acetate buffer $(\mathrm{pH} \mathrm{4.8})$ in 6.3, 10.7, 15.3 and $30.7 \mathrm{~min}$ after depositing $0.90 \mathrm{ng}$ of the cellulases. Their 3D height maps are available in Figure S2. (b) Change of a cross-sectional profile of the pit as a function of reaction time. (c and $\mathbf{d})$ Schematic illustration showing quantification of the pit by measuring cross-sectional profile (c) and volume (d). White lines represent cellulose nanofibers, and blue areas represent the buffered solution that filled up pores of the nanofibrous cellulose matrix. The cross-sectional profile (thick red curve in c) can be used to evaluate shape and 
dimension of the pit, but does not give information necessary to assay enzymatic reaction. In contrast, the pit volume (pink area in d) allows calculating the mass of the hydrolyzed cellulose nanofibers (thick white lines in $\mathbf{d}$ ), by multiplying it with the fiber density. (e) Change of the hydrolyzed mass of cellulose and the volume of the pit formed at room temperature after depositing 0.18 (black), 0.36 (blue), 0.72 (red), and $0.90 \mathrm{ng}$ (green) of the cellulases. Each data points represent average values of at least 5 independent runs, and vertical error bars represent standard deviation. Horizontal error bars represent scan times necessary to obtain 3D height maps (approximately $5 \mathrm{~min}$ ). Broken lines are linear fits to the first three data points. (f) Relationship between measured hydrolysis rate and the amount of deposited cellulases.

\section{Figure 3 | Recognizing microbial production of cellulases by surface}

pitting. (a) 3D topographic image of a colony of Escherichia coli formed on the surface of a nanofibrous cellulose matrix containing Luria-Bertani medium. The image was obtained after incubation at $37^{\circ} \mathrm{C}$ for $9 \mathrm{~h}$. Its 3D height map with a scale bar is available in Figure S3. (b-d) 3D topographic images of a pit formed by $S$. degradans on a nanofibrous cellulose matrix $\left(0.03 \mathrm{~g} \cdot \mathrm{cm}^{-3}\right.$ cellulose $)$ after incubation at room temperature for (b) $24 \mathrm{~h},(\mathbf{c}) 30 \mathrm{~h}$, and (d) $48 \mathrm{~h}$. Their 3D height maps with scale bars are available in Figure S3. (e) Change of a crosssectional profile of the pit as a function of incubation time. (f) Change of depth and width (full width at half depth) of the pit as a function of incubation time. Both increased linearly at similar rates with the incubation time, suggesting isotropic growth of the pit. (g) Changes in the hydrolyzed mass of cellulose and volume of pits as a function of incubation time. Red points represent data obtained by analyzing the pit in $\mathbf{e}$. Blue points represents data from a separate measurement to estimate variation of the results. (h) Optical micrograph showing the surface of a nanofibrous cellulose plate during initial screening 
from Okinawa Trough. Cellulolytic bacteria formed pits (indicated by red triangles) and could be separated easily from non-cellulolytic ones that formed normal colonies of different sizes. Image was taken after incubation at $20^{\circ} \mathrm{C}$ for $15 \mathrm{~d}$. Scale bar represents $1 \mathrm{~mm}$. (i) SEM image showing a novel deep-sea bacterium (strain GE09) growing in a pit on a nanofibrous cellulose plate $(0.01$ $\mathrm{g}^{\circ} \mathrm{cm}^{-3}$ cellulose) after incubation at $20^{\circ} \mathrm{C}$ for $6 \mathrm{~d}$. The bottom part shows GE09 cells, and the upper part shows nanofibrous cellulose.

\section{Figure 4 | Predicted carbohydrate active enzyme (CAZyme) genes from the} deep-sea bacteria GE09 and the characteristics of its cellulases. (a)

Predicted genes encoding CAZy domains with the type I or II secretion signals in the genomes of GE09, T. turnerae and S. degradans. The numbers of total sequences were shown at the center of each chart. (b) Phylogenetic tree of $\mathrm{GH}$ family 6 domain of all entries in the CAZy database and of five sequences from GE09. Branches of GE09 sequences was indicated as branch a and b, colored in blue. Abbreviations and representative sequences: EG, endo-glucanase; CBH, cellobiohydrolase; 1, Thermobifida (T.) fusca Cel6A; 2, Cellulomonas fimi Cel6A; 3, Ciona savignyi cellulose synthase; 4, Trichoderma reesei Cel6A; 5, Humicola insolens Cel6B; 6, Orpinomyces sp. CelC7; 7, Xanthomonas oryzae CbsA; 8, Cellulomonas fimi Cel6B; 9, Teredinibacter turnerae CelAB, Saccharophagus degradans Cel6A; 10, T. fusca Cel6B. A modeled structure of the cellulase of GE09 (MARGE09_P1668) and the structure of the T. fusca Cel6B (Protein Data Bank accession code 4B4F) are shown in right panel. Both models are shown with cellohexaoses from 4B4F (green) at their ligand binding sites. Loops adjacent to the binding sites and inserted loop regions were colored in magenta and blue, respectively. (c) Unknown modular structures of cellulases. GH family 6 domains with dotted line lack essential catalytic 
residues. Abbreviations: Spll, type II secretion signal; PKD, polycystic kidney disease; CIP1, cellulose induced protein I; TSP3, thrombospondin type 3. 